\title{
Sex differences in outcomes of methadone maintenance treatment for opioid use disorder: a systematic review and meta-analysis
}

\author{
Monica Bawor BSc, Brittany B. Dennis BA, Anuja Bhalerao, Carolyn Plater MSW, Andrew Worster MD, \\ Michael Varenbut MD, Jeff Daiter MD, David C. Marsh MD, Dipika Desai MSc, Meir Steiner MD PhD, \\ Rebecca Anglin MD PhD, Guillaume Pare MD MSc, Lehana Thabane PhD, Zainab Samaan MBChB PhD
}

\section{Abstract}

Background: Opioid use disorder is a serious international concern with limited treatment success. Men and women differ in their susceptibility to opioid use disorder and response to methadone treatment and can therefore benefit from sex-specific treatment. We performed a systematic review of the literature on outcomes of methadone maintenance treatment for opioid use disorder in men and women related to drug use, health status and social functioning.

Methods: We searched PubMed, Embase, PsycINFO and CINAHL for observational or randomized controlled studies involving adults 18 years of age or older undergoing methadone treatment for opioid use disorder. Studies were included if they investigated sex differences in methadone treatment outcomes. Two authors independently reviewed and extracted data. Meta-analyses were performed when possible; risk of bias and quality of evidence were also assessed.

Results: Twenty studies with 9732 participants were included, of which 18 were observational and 2 were randomized controlled trials. Men and women differed significantly in alcohol use (odds ratio [OR] 0.52, 95\% confidence interval [Cl] 0.31 to 0.86 ), amphetamine use (OR $1.47,95 \% \mathrm{Cl} 1.12$ to 1.94 ), legal involvement (OR $0.63,95 \% \mathrm{Cl} 0.47$ to 0.84 ) and employment during treatment (OR 0.39 , $95 \% \mathrm{Cl} 0.21$ to 0.73 ). Opioid use patterns were similar among men and women. Risk of bias was moderate, and quality of evidence was generally low.

Interpretation: Sex differences were evident in polysubstance use, legal involvement and employment status among men and women receiving methadone treatment for opioid use disorders. Although the quality of evidence was low, our review highlights the need for improved implementation of sex-specific treatment strategies.

anadians have recently surpassed US citizens to become the highest opioid analgesic consumers in the world. ${ }^{1}$ In 2012, CMA7 published a report showing that about 200000 Canadians regularly use prescription opioids, ${ }^{2}$ which are becoming the most commonly used drugs of abuse. ${ }^{3}$ There has been a surge in opioid prescriptions of $150 \%$ over the last decade. ${ }^{4}$ As a result, the number of hospital admissions and deaths due to opioid use and overdose has grown substantially. ${ }^{5}$ In addition to the collective health care costs, each untreated case of opioid addiction has a social cost of Can\$45 000 annually per person, ${ }^{6}$ a major economic outlay.

Currently, there are about 35000 patients receiving substitute opioid therapy with methadone at addiction treatment centres in Ontario. ${ }^{7}$ Several maintenance treatment programs are available, with methadone being the most commonly prescribed treatment for opioid use disorder. ${ }^{8}$ Effectiveness rates of $20 \%-$
$70 \%$ for methadone maintenance treatment are reported in the literature. ${ }^{9-12}$ Treatment response in opioid use disorder is difficult to define and has been broadly described in the literature, which makes clinical interpretation of these studies challenging. There are no agreed-on criteria that characterize a treatment as a success or failure; therefore, there is no accurate way to know whether treatment is working or whether the health care resources invested in treatment are producing any benefit.

Competing interests: None declared.

This article has been peer reviewed.

Correspondence to: Zainab Samaan, samaanz@mcmaster.ca CMAJ Open 2015. DOI:10.9778/cmajo.20140089 
There is evidence, however, of high variability in response to methadone treatment between patients, ${ }^{13}$ which indicates that patients may have different treatment needs. Men and women especially are known to differ in addiction susceptibility and behaviour, including age at first opioid use, progression to regular use and age at treatment entry. ${ }^{14-16}$ It is also likely that men and women differ in methadone treatment outcomes, although these differences are unclear in the literature. Hence, current treatment standards that offer the same clinical management of opioid use disorder for women and men may not achieve optimum treatment outcomes for both sexes.

We performed a systematic review of the literature on sex differences in methadone treatment outcomes related to drug use behaviour, health status and social functioning, using a meta-analysis where possible. We critically evaluated the evidence and highlighted areas for future research. Our aim was to identify possible sex-specific patient needs that can be addressed with an individualized treatment strategy to produce improved treatment outcomes, increased treatment efficacy and decreased risk of adverse events.

\section{Methods}

This review is reported in accordance with the Preferred Reporting Items for Systematic Reviews and Meta-Analyses guidelines $^{17}$ (completed checklist in Appendix 1, www.cmajopen .ca/content/3/3/E344/suppl/DC1). The review has been registered with PROSPERO (no. CRD42013006549), and the detailed methods have been previously reported in a protocol. ${ }^{18}$

\section{Literature search}

We searched PubMed/MEDLINE, Embase, PsycINFO and CINAHL databases from inception to Aug. 11, 2014, for relevant articles. The following search terms and their $\mathrm{MeSH}$ subject headings were used: "opioid-related disorders"; "opiate substitution treatment"; "methadone maintenance"; "sex differences/characteristics"; "gender differences"; "sex"; "male"; "female"; "men"; and "women." No language or time constraints were applied; however, the search was limited to people aged 18 years or older. The complete search strategy is found in the published protocol. ${ }^{18}$ We included completed, published and peer-reviewed studies to ensure that we captured only high-quality evidence; we did not screen the grey literature. Included studies were supported primarily by national funding agencies, and no conflicts of interest were declared.

\section{Study selection}

We included observational studies and randomized controlled trials (RCTs) that focused on sex differences among patients receiving methadone treatment for opioid use disorders. In this review, "opioid use disorder" encompasses all classifications of opioid-related disorders set forth by the Diagnostic and Statistical Manual of Mental Disorders that have been used to date (i.e., abuse, dependence and addiction).

We included studies if their primary focus was sex differences in methadone treatment outcomes, including treatment response, retention, remission status after treatment, polysubstance abuse, methadone dosage, drug-related adverse events, health status, psychological status, death, criminal activity, highrisk sexual behaviour, social support and employment. Although marital status is not a direct outcome of methadone treatment, it is worthwhile to examine this variable. Having a partner can be indicative of a stable and supportive environment for the patient, which can positively influence the recovery process. ${ }^{19,20}$

We were interested in all types of patients, regardless of ethnicity or geographic location. We excluded studies involving patients receiving an opioid substitution treatment other than methadone or using methadone for the treatment of chronic pain.

\section{Data collection}

Two of us (M.B. and A.B.) independently reviewed articles at each screening stage; disagreements were resolved by consensus. We extracted data in duplicate using a pilot-tested data extraction form. ${ }^{18}$ Studies not meeting our inclusion criteria were excluded and reasons for exclusion recorded. We obtained information on study characteristics and design, sample population and methadone treatment outcomes.

\section{Risk of bias assessment}

For observational studies, 2 authors (M.B. and A.B.) independently assessed risk of bias using a version of the NewcastleOttawa Scale ${ }^{21}$ that was modified for this review. We assessed risk of bias using the Cochrane Collaboration's tool for RCTs. ${ }^{22}$ We applied the Grading of Recommendations Assessment, Development and Evaluation (GRADE) framework $^{23}$ to the meta-analyses to evaluate the confidence in the summarized evidence.

\section{Data synthesis}

We used a Mantel-Haenszel random-effects model for the summary estimate, assuming heterogeneity between studies. We computed a pooled odds ratio (OR) for dichotomous outcomes and standardized mean difference for continuous outcomes. Summary measures are presented with corresponding $95 \%$ confidence intervals (CIs). We assessed heterogeneity using the $I^{2}$ statistic. Publication bias was evaluated using funnel plots (Review Manager [RevMan] version 5.3, The Cochrane Collaboration). We performed analyses using RevMan version 5.3.

\section{Results}

\section{Search results and study characteristics}

We included 20 studies with 9732 participants in the review (Figure 1). ${ }^{24-43}$ The strength of agreement between the 2 independent raters was high for title screens ( $\mathrm{k}$ value $=0.823,95 \%$ CI 0.736 to 0.910 ), abstract screens (к value $=0.898,95 \%$ CI 0.760 to 1.000$)$ and full-text screens ( $\mathrm{k}$ value $=0.834,95 \%$ CI 0.615 to 1.000$)$.

We included 18 cohort studies and 2 RCTs. The studies were conducted in the United States (16), Israel (2), Spain (1) and Sweden (1). The sample size for each study varied from 53 to 2683 participants, and the studies included mostly men. 
The most frequently reported ethnicities were white, black and Hispanic. Characteristics of the 20 studies are presented in Table 1.

\section{Risk of bias assessment}

We evaluated bias (selection, performance, detection and information) for all of the studies. Generally, risk of bias was moderate to high for the observational studies (Appendix 2 [Table A] www.cmajopen.ca/content/3/3/E344/suppl/DC1) and low for RCTs (Appendix 2 [Table B]).

\section{Sex differences in methadone treatment outcomes}

\section{Substance use}

Eleven studies focused on polysubstance use during treatment (Table 1). We performed a separate meta-analysis for each substance reported: alcohol, amphetamines, benzodiazepines, cannabis and cocaine.

The odds of self-reporting alcohol use while receiving methadone treatment were significantly lower among women than among men ${ }^{24,30,39}$ (OR 0.52, 95\% CI 0.31 to 0.86 ) (Table 2, Figure 2). The odds of amphetamine use while receiving methadone treatment were significantly greater among women than among men $^{35,38}$ (OR 1.47, 95\% CI 1.12 to 1.94) (Table 2, Figure 3).

No significant sex differences were seen in the use of opioids or other substances while receiving methadone treatment (see Appendix 2 [Figures A, C-E] for respective forest plots for opioid, benzodiazepine, cannabis and cocaine use), in treatment retention (Appendix 2 [Figure B]) or in methadone dosage (Appendix 2 [Figure F]).

\section{Health status}

As per the protocol, ${ }^{18}$ we intended to analyze health outcomes, including methadone-related adverse events, health and psychological status; however, data on these outcomes were unsuitable for a meta-analysis.

\section{Social functioning}

Women were less likely to report arrests or legal supervision (including probation or parole) during treatment than were $\operatorname{men}^{24,30}$ (OR 0.63, 95\% CI 0.47 to 0.84) (Table 2, Figure 4). Women were also less likely than men to be employed $24,25,28,30,39$ (OR 0.39, 95\% CI 0.21 to 0.73) (Table 2, Figure 5). Studies that measured high-risk sexual behaviour had highly variable outcome definitions, which precluded a meta-analysis. No significant differences were found in marital status (married or common-law) between men and women during methadone treatment ${ }^{24,25,30,39}$ (Table 2, Appendix 2 [Figure G]).

\section{Long-term prognosis}

Six studies assessed outcomes of long-term methadone maintenance treatment. Patients were followed longitudinally or were identified retrospectively, with follow-up periods varying from 1 to 25 years after treatment completion. These studies provided data on several treatment-related outcomes, including illicit opioid use (5 studies), legal involvement (2), employment (2) and death (3). Owing to large differences in follow-up periods, a meta-analysis was not suitable; we therefore provide a brief summary of the findings.

Jimenez-Treviño and colleagues ${ }^{31}$ found that, 25 years after completion of treatment, the proportion of men using heroin was significantly greater than that of women $(32.5 \% \mathrm{v}$. $0 \%, p=0.04$ ). In the remaining 4 studies, no significant sex differences were found in illicit opioid use during 4 weeks ${ }^{24}$ or 1 year ${ }^{28,33,37}$ of follow-up.

Both Marsh and Simpson ${ }^{33}$ and Savage and Simpson ${ }^{37}$ found a greater proportion of men than women reporting lifetime arrest or incarceration ( $>3$ days) at follow-up (30\% v. $12 \%, p<0.05$ and $27 \%$ v. $15 \%, p<0.05$, respectively). The proportion of men reporting employment (> 6 months) during the first year after treatment or after 1 year of followup was also significantly greater than that of women ${ }^{33,37}$ $(51 \%$ v. $31 \%, p<0.05$ and $68 \%$ v. $41 \%, p<0.05)$. Risk of death at 1 year did not differ significantly between men and

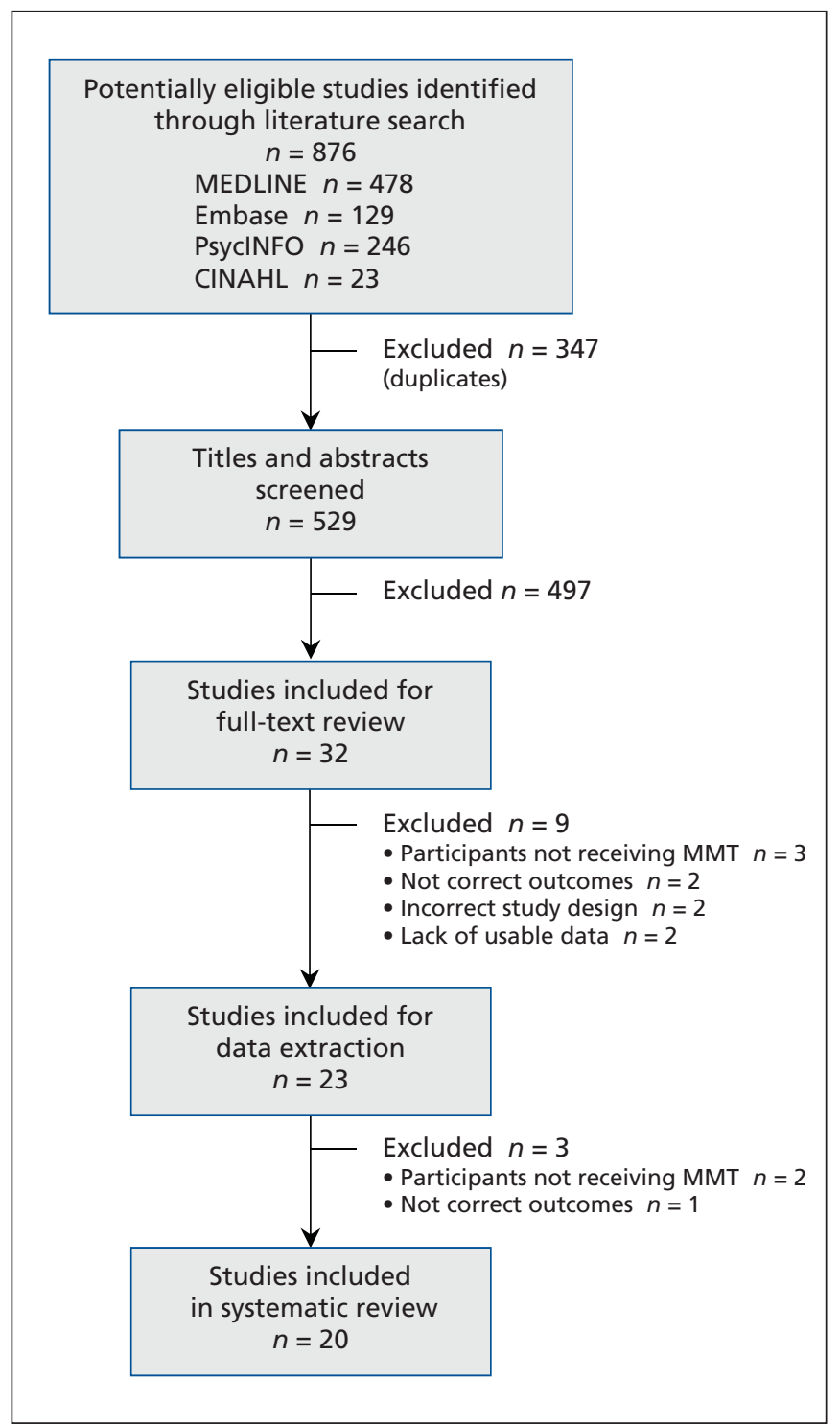

Figure 1: Selection of studies for inclusion in the systematic review and meta-analyses. MMT = methadone maintenance treatment. 


\section{OPEN}

Research

Table 1: Characteristics of the $\mathbf{2 0}$ studies included in the systematic review

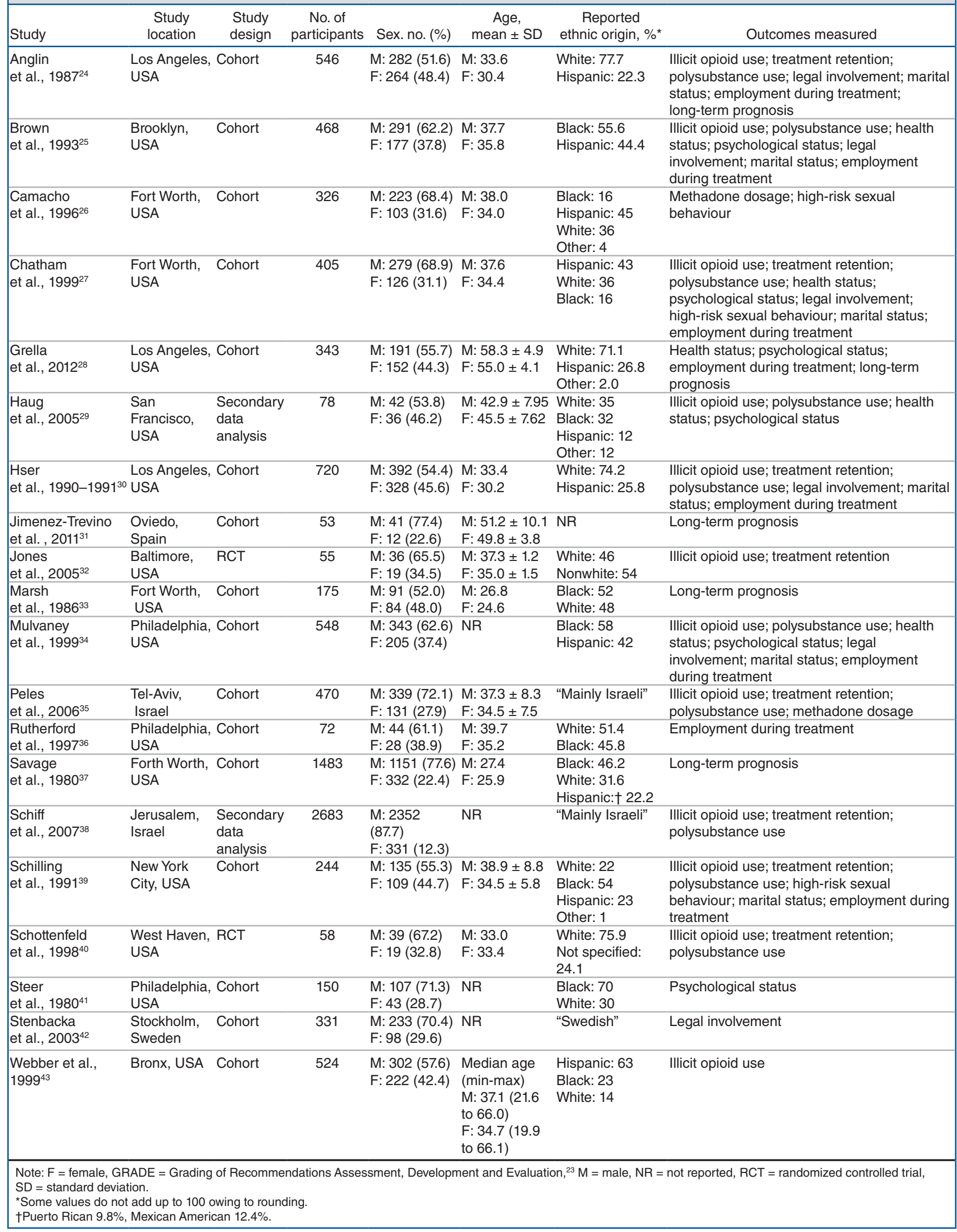


Table 2: Summary of results of meta-analysis to identify sex differences among the included studies

\begin{tabular}{|c|c|c|c|c|c|c|c|}
\hline \multirow[b]{2}{*}{ Outcome } & \multirow{2}{*}{$\begin{array}{l}\text { No. of } \\
\text { studies }\end{array}$} & \multicolumn{2}{|c|}{ Subjects, $n$} & \multirow{2}{*}{$\begin{array}{l}\text { Pooled OR or SMD } \\
\qquad(95 \% \mathrm{Cl})\end{array}$} & \multirow[b]{2}{*}{$I^{2}, \%$} & \multirow{2}{*}{$\begin{array}{l}\text { Summary of } \\
\text { sex differences }\end{array}$} & \multirow{2}{*}{$\begin{array}{l}\text { GRADE quality } \\
\text { of evidence }\end{array}$} \\
\hline & & M & $\mathrm{F}$ & & & & \\
\hline \multicolumn{8}{|l|}{ Illicit opioid use } \\
\hline Cohort studies $24,30,43$ & 3 & 976 & 814 & 0.81 (0.50 to 1.31$)$ & 82 & - & Very low ${ }^{*} \dagger$ \\
\hline $\mathrm{RCTs}^{32,40^{*} 40 \dagger}$ & $3 \ddagger$ & 75 & 38 & $1.39(0.61$ to 3.19$)$ & 0 & - & Moderate§ \\
\hline Treatment retention ${ }^{27,30,35}$ & 3 & 1010 & 585 & 1.01 (0.62 to 1.63$)$ & 77 & - & Low \\
\hline \multicolumn{8}{|l|}{ Polysubstance use } \\
\hline Alcohol use $24,30,39$ & 3 & 809 & 701 & $0.52(0.31$ to 0.86$)$ & 77 & $\begin{array}{l}\text { Women less } \\
\text { likely to use } \\
\text { alcohol }\end{array}$ & Moderate* \\
\hline Amphetamine use $\mathrm{e}^{35,38}$ & 2 & 2691 & 462 & $1.47(1.12$ to 1.94$)$ & 0 & $\begin{array}{l}\text { Women more } \\
\text { likely to use } \\
\text { amphetamines }\end{array}$ & Low \\
\hline Benzodiazepine use ${ }^{35,38}$ & 2 & 2691 & 462 & 0.94 (0.70 to 1.27$)$ & 44 & - & Low \\
\hline Cannabis use $\mathrm{e}^{35,38}$ & 2 & 2691 & 462 & 0.85 (0.67 to 1.08$)$ & 0 & - & Low \\
\hline Cocaine use $\mathrm{e}^{35,38,39}$ & 3 & 2826 & 571 & 1.07 (0.64 to 1.78$)$ & 76 & - & Very low*†§I \\
\hline \multicolumn{8}{|l|}{ Other } \\
\hline 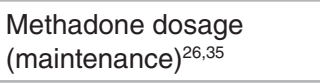 & 2 & 562 & 234 & $-2.38(-5.67$ to 0.91$)$ & 0 & - & Low \\
\hline Legal involvement ${ }^{24,30}$ & 2 & 674 & 592 & 0.63 (0.47 to 0.84$)$ & 39 & $\begin{array}{l}\text { Women less } \\
\text { likely to report } \\
\text { arrests or legal } \\
\text { supervision }\end{array}$ & Moderate ${ }^{*}+\emptyset$ \\
\hline $\begin{array}{l}\text { Employment during } \\
\text { treatment }{ }^{24,25,28,30,39}\end{array}$ & 5 & 1291 & 1030 & 0.39 (0.21 to 0.73$)$ & 91 & $\begin{array}{l}\text { Women less } \\
\text { likely to be } \\
\text { employed }\end{array}$ & Moderate* $\mathbb{I}^{\star *}$ \\
\hline Marital status ${ }^{24,25,30,39}$ & 4 & 1100 & 878 & $0.96(0.75$ to 1.21$)$ & 0 & - & Low \\
\hline Death $^{27,43}$ & 2 & 581 & 353 & $1.61(0.60$ to 4.33$)$ & 83 & - & Low \\
\hline \multicolumn{8}{|c|}{ 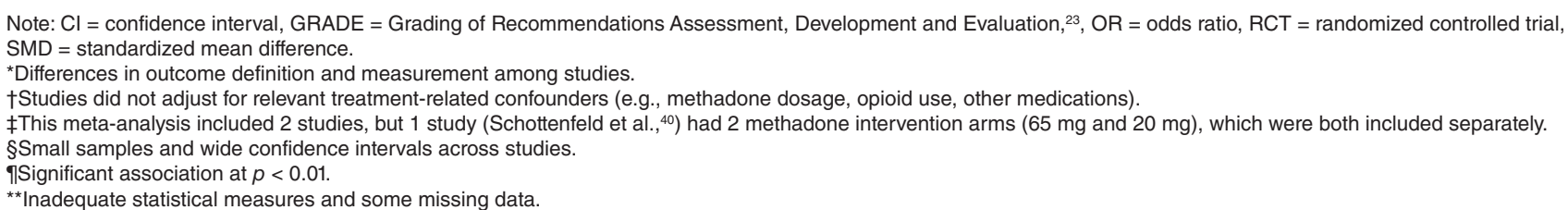 } \\
\hline
\end{tabular}

\begin{tabular}{|c|c|c|c|}
\hline \multirow[b]{2}{*}{ Study } & \multicolumn{2}{|c|}{ Events, $n / N$} & \multirow[b]{2}{*}{ OR $(95 \% \mathrm{Cl})$} \\
\hline & Women & Men & \\
\hline Anglin et al. ${ }^{24}$ & $45 / 264$ & $89 / 282$ & 0.45 (0.30 to 0.67$)$ \\
\hline Hser et al. ${ }^{30}$ & $46 / 328$ & $122 / 392$ & $0.36(0.25$ to 0.53$)$ \\
\hline Schilling et al. ${ }^{39}$ & $60 / 109$ & $77 / 135$ & 0.92 (0.55 to 1.53$)$ \\
\hline Overall & $151 / 701$ & $288 / 809$ & 0.52 (0.31 to 0.86$)$ \\
\hline Heterogeneity: $I^{2}$ & & & \\
\hline
\end{tabular}

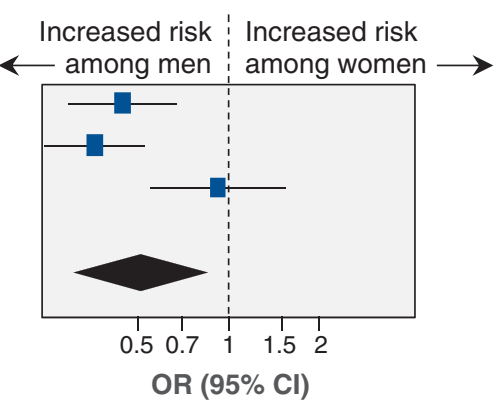

Figure 2: Meta-analysis of sex-related difference in alcohol use over the past 6 months of treatment among women and men receiving methadone maintenance treatment for opioid use disorders. An odds ratio (OR) less than 1.0 indicates an increased likelihood of men reporting alcohol use. $\mathrm{Cl}=$ confidence interval. 
women $^{27,43}$ (OR 1.61, 95\% CI 0.60 to 4.33) (Table 2, Appendix 2 [Figure H]). Jimenez-Treviño and colleagues ${ }^{31}$ also found no significant difference in mortality between men and women at 25 years.

\section{Interpretation}

In this review, we have summarized the results from 20 studies on sex differences in methadone treatment outcomes. We found that women were less likely than men to use alcohol, report arrests or legal supervision and be employed during treatment. However, women were more likely than men to use amphetamines during treatment.

Our findings are consistent with the trends observed in past individual studies of patients with opioid use disorder and those in methadone treatment. ${ }^{14-16,24,44}$ They are also in line with traditional sex-role expectations (e.g., higher unemployment among women, more alcohol use among men).

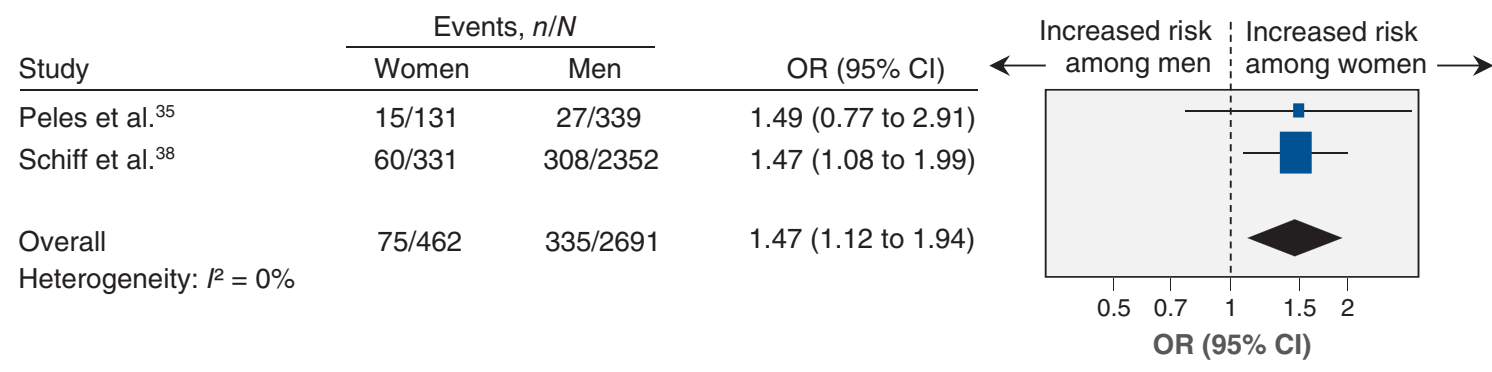

Figure 3: Meta-analysis of sex-related difference in amphetamine use over the past 6 months of treatment among women and men receiving methadone maintenance treatment for opioid use disorders. An odds ratio (OR) greater than 1.0 indicates an increased likelihood of women reporting amphetamine use. $\mathrm{Cl}=$ confidence interval.

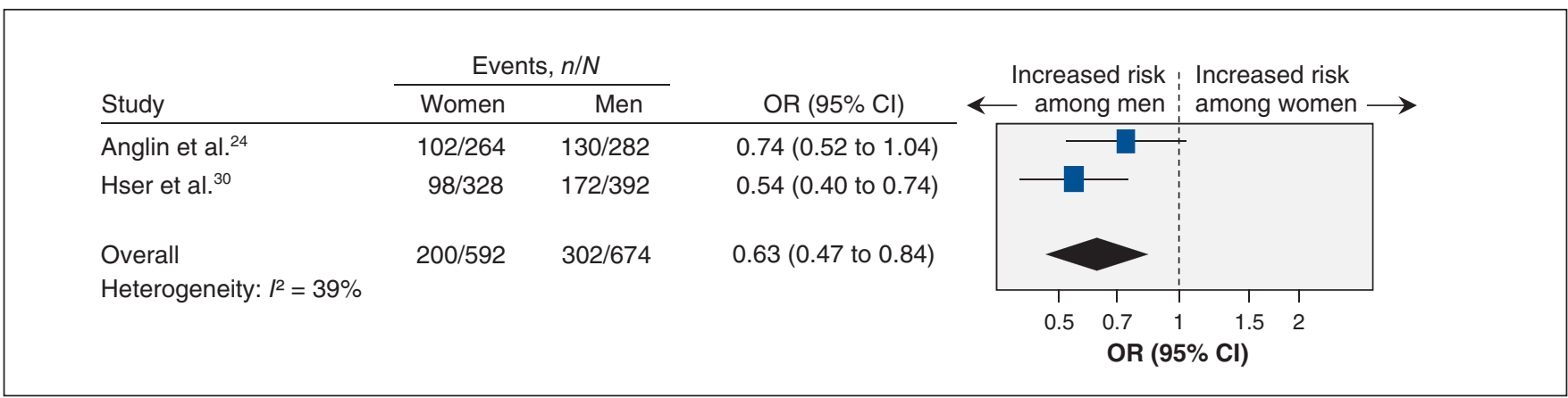

Figure 4: Meta-analysis of sex-related difference in self-reported legal status during treatment among women and men receiving methadone maintenance treatment for opioid use disorders. An odds ratio (OR) less than 1.0 indicates an increased likelihood of men reporting arrests or legal supervision (e.g., probation or parole). $\mathrm{Cl}=$ confidence interval.

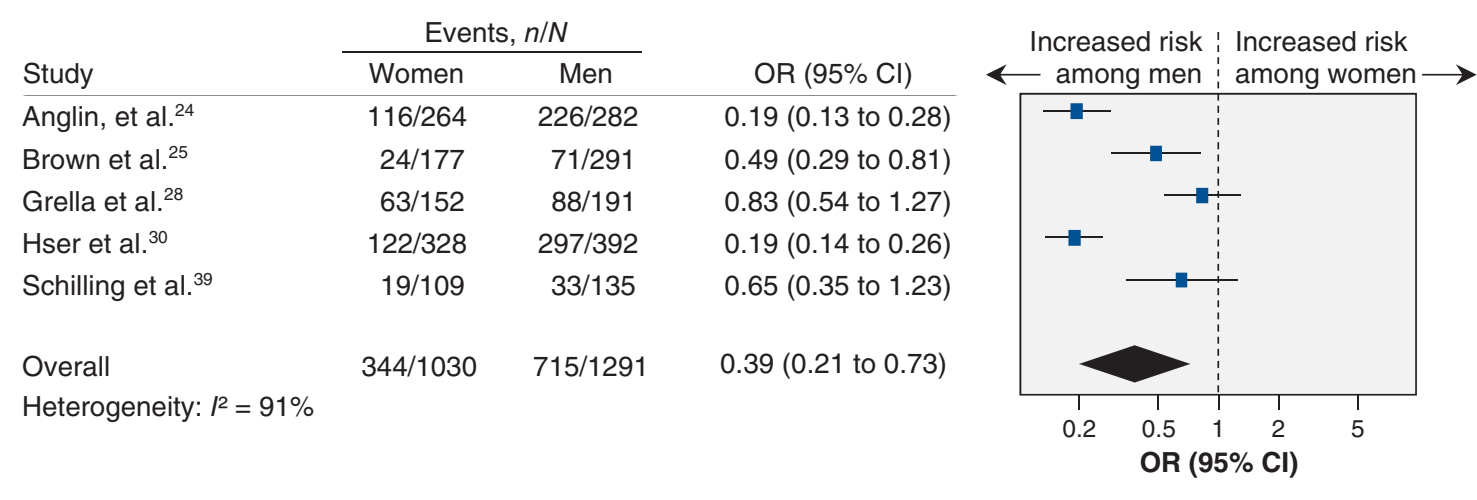

Figure 5: Meta-analysis of sex-related difference in self-reported employment status during treatment among women and men receiving methadone maintenance treatment for opioid use disorders. An odds ratio (OR) less than 1.0 indicates an increased likelihood of men being employed. $\mathrm{Cl}=$ confidence interval. 


\section{Limitations}

The current literature on methadone treatment outcomes lacks common definitions or standard measurements for treatment response, and what constitutes a good or poor treatment response remains unclear. We included a comprehensive list of outcomes to account for this variation in definitions. As well, the differences in outcome measurements made it impossible to combine the results of all studies, and several studies were not suitable for meta-analyses. . $^{29,31,33,34,36,37,41,42}$ As a result, individual meta-analyses in this review contained, at most, 5 studies, thus providing limited generalizability.

Sex differences in this review may actually be a representation of the general population and not be specific to patients receiving methadone treatment. For instance, the association between men and criminal activity is seen in the general population ${ }^{45}$ and therefore may not be directly attributable to opioid use disorder or methadone treatment. We were also unable to establish any causality in outcomes because the data were cross-sectional. Thus, we cannot draw conclusions regarding possible significant improvements in outcomes between treatment initiation and completion.

The nature of the treatment interventions was poorly described, and there were differences in treatment practices among $\mathrm{RCTs}^{32,40}$ and among studies conducted in private methadone programs. ${ }^{25,34}$ However, most studies had comparable methadone treatment practices, and specific outcomes were consistent across studies.

We assessed risk of bias and overall quality of evidence to evaluate our confidence in our findings. Most studies were at moderate to high risk for bias, in several cases owing to small or unrepresentative samples, failure to adjust for confounders and lack of objective outcome assessment. The overall quality of evidence was low to moderate, most likely because of differences in outcome measurement between studies, allowing for high variation and heterogeneity.

\section{Implications for practice and research}

Using an extensive list of outcomes, this review has shown how men and women differ in their response to methadone treatment. These findings may be useful to inform the design of existing sex-specific treatment programs or the development of comprehensive sex-specific patient-centred treatment models in areas where they have not yet been introduced. Programs are encouraged to incorporate medical care, other substance-use treatment programs (e.g., alcohol or amphetamine abuse), counselling, mental health services and employment needs into their treatment plans, since these services are known to be associated with improved outcomes. ${ }^{46}$

The current program standards and clinical guidelines for methadone maintenance treatment issued by the College of Physicians and Surgeons of Ontario ${ }^{47}$ were developed from individual study findings and are therefore not informed through systematic summaries of the evidence. The findings of our systematic review may be useful in informing development and updates of best-practice guidelines. However, more methodologically sound studies are required to provide a better understanding and characterization of sex differences in the use of methadone treatment in opioid use disorders, for health care providers, health policy-makers and patients.

With additional high-quality studies, a more conclusive record of sex differences in methadone treatment could be developed. Subsequent studies could assess the efficacy of sexspecific treatment and determine which approaches lead to positive outcomes. Moreover, an investigation into potential explanations for sex differences - whether they are biologically or socially driven - is needed so that these factors can also be incorporated into treatment. It would also be of interest to assess knowledge of sex differences within methadone clinics and ways in which awareness of these differences could be heightened. Furthermore, we do not know which outcomes are important to patients themselves. Lastly, the costeffectiveness of integrating specialized treatment for men and women should be assessed, because implementation of these strategies would likely reduce overall costs, treatment-related and otherwise.

\section{Conclusion}

Sex differences were evident in polysubstance use, legal involvement and employment status among men and women receiving methadone treatment for opioid use disorders. More studies are needed to better elucidate the presence of sex differences in methadone treatment outcomes. Studies need to be replicated in larger samples, using standardized assessments and measures, and with appropriate statistical testing to improve our understanding of sex differences and draw appropriate conclusions that can be applied in the clinical setting. With further research, it is our hope that these findings can be helpful in improving treatment for patients with opioid use disorders and the overall field of research in opioid addiction.

\section{References}

1. World drug report 2014. New York: United Nations Office on Drugs and Crime; 2014. Available: www.unodc.org/documents/wdr2014/World_Drug_ Report_2014_web.pdf (accessed 2015 Aug. 11).

2. Webster PC. Medically induced opioid addiction reaching alarming levels. CMA7 2012;184:285-6.

3. Fischer B, Rehm J, Goldman B, et al. Non-medical use of prescription opioids and public health in Canada: an urgent call for research and interventions development. Can 7 Public Health 2008;99:182-4.

4. Manchikanti L, Helm SII, Fellows B, et al. Opioid epidemic in the United States. Pain Physician 2012;15(Suppl):ES9-38.

5. World drug report 2012. New York: United Nations Office on Drugs and Crime; 2012. Available: www.unodc.org/unodc/en/data-and-analysis/WDR -2012.html (accessed 2015 Aug. 11).

6. Wall R, Rehm J, Fischer B, et al. Social costs of untreated opioid dependence. 7 Urban Health 2000;77:688-722.

7. Methadone. Toronto: Centre for Addiction and Mental Health; 2010. Available: www.camh.ca/en/hospital/health_information/a_z_mental_health_and_addiction _information/methadone/Pages/methadone.aspx (accessed 2015 Aug. 11).

8. Fischer B. Prescriptions, power and politics: the turbulent history of methadone maintenance in Canada. F Public Health Policy 2000;21:187-210.

9. Dutta R, Roy S. Mechanism(s) involved in opioid drug abuse modulation of HAND. Curr HIV Res 2012;10:469-77.

10. Eap CB, Buclin T, Baumann P. Interindividual variability of the clinical pharmacokinetics of methadone: implications for the treatment of opioid dependence. Clin Pharmacokinet 2002;41:1153-93.

11. Mattick RP, Breen C, Kimber J, et al. Methadone maintenance therapy versus no opioid replacement therapy for opioid dependence. Cochrane Database Syst Rev 2009; (3):CD002209.

12. Oviedo-Joekes E, Guh D, Brissette S, et al. Effectiveness of diacetylmorphine versus methadone for the treatment of opioid dependence in women. Drug Alcohol Depend 2010;111:50-7. 
13. Li Y, Kantelip JP, Gerritsen-van Schieveen P, et al. Interindividual variability of methadone response: impact of genetic polymorphism. Mol Diagn Ther 2008;12:109-24.

14. Anglin MD, Hser YI, McGlothlin WH. Sex differences in addict careers. 2. Becoming addicted. Am 7 Drug Alcobol Abuse 1987;13:59-71.

15. Hser YI, Anglin MD, McGlothlin W. Sex differences in addict careers. 1. Initiation of use. Am 7 Drug Alcohol Abuse 1987;13:33-57.

16. Hser YI, Anglin MD, Booth MW. Sex differences in addict careers. 3. Addiction. Am 7 Drug Alcohol Abuse 1987;13:231-51.

17. Moher D, Liberati A, Tetzlaff J, et al. Preferred reporting items for systematic reviews and meta-analyses: the PRISMA statement. PLoS Med 2009;6:e1000097.

18. Bawor M, Dennis BB, Anglin R, et al. Sex differences in outcomes of methadone maintenance treatment for opioid addiction: a systematic review protocol. Syst Rev 2014;3:45.

19. Eldred CA, Washington MN. Interpersonal relationships in heroin use by men and women and their role in treatment outcome. Int 7 Addict 1976;11:117-30.

20. Gerra G, Ferri M, Polidori E, et al. Long-term methadone maintenance effectiveness: psychosocial and pharmacological variables. F Subst Abuse Treat 2003; 25:1-8.

21. Wells G, Shea B, O'Connell D, et al. The Newcastle-Ottawa Scale (NOS) for assessing the quality of nonrandomised studies in meta-analyses. Ottawa: Ottawa Hospital Research Institute; 2000.

22. Higgins JP, Altman DG, Gotzsche PC, et al. The Cochrane Collaboration's tool for assessing risk of bias in randomised trials. BM7 2011;343:d5928.

23. Guyatt GH, Kunz R, Brozek J, et al. GRADE guidelines 6. Rating the quality of evidence - imprecision. 7 Clin Epidemiol 2011;64:1283-93.

24. Anglin MD, Hser YI, Booth MW. Sex differences in addict careers. 4. Treatment. Am 7 Drug Alcobol Abuse 1987;13:253-80.

25. Brown LS Jr, Alterman AI, Rutherford MJ, et al. Addiction Severity Index scores of four racial/ethnic and gender groups of methadone maintenance patients. 7 Subst Abuse 1993;5:269-79.

26. Camacho LM, Bartholomew NG, Joe GW, et al. Gender, cocaine and duringtreatment HIV risk reduction among injection opioid users in methadone maintenance. Drug Alcobol Depend 1996;41:1-7.

27. Chatham LR, Hiller ML, Rowan-Szal GA, et al. Gender differences at admission and follow-up in a sample of methadone maintenance clients. Subst Use Misuse 1999;34:1137-65.

28. Grella CE, Lovinger K. Gender differences in physical and mental health outcomes among an aging cohort of individuals with a history of heroin dependence. Addict Behav 2012;37:306-12.

29. Haug NA, Sorensen JL, Lollo ND, et al. Gender differences among HIV-positive methadone maintenance patients enrolled in a medication adherence trial. AIDS Care 2005;17:1022-9.

30. Hser YI, Anglin MD, Liu Y. A survival analysis of gender and ethnic differences in responsiveness to methadone maintenance treatment. Int 7 Addict 1990-1991;25(11a):1295-315.

31. Jimenez-Treviño L, Saiz PA, García-Portilla MP, et al. A 25-year follow-up of patients admitted to methadone treatment for the first time: mortality and gender differences. Addict Behav 2011;36:1184-90.

32. Jones HE, Fitzgerald H, Johnson RE. Males and females differ in response to opioid agonist medications. Am 7 Addict 2005;14:223-33.

33. Marsh KL, Simpson DD. Sex differences in opioid addiction careers. Am 7 Drug Alcohol Abuse 1986;12:309-29.

34. Mulvaney FD, Brown LS Jr, Alterman AI, et al. Methadone-maintenance outcomes for Hispanic and African-American men and women. Drug Alcohol Depend 1999;54:11-8.

35. Peles E, Adelson M. Gender differences and pregnant women in a methadone maintenance treatment (MMT) clinic. 7 Addict Dis 2006;25:39-45.

36. Rutherford MJ, Cacciola JS, Alterman AI, et al. Social competence in opiateaddicted individuals: gender differences, relationship to psychiatric diagnoses, and treatment response. Addict Behav 1997;22:419-25.

37. Savage LJ, Simpson DD. Posttreatment outcomes of sex and ethnic groups treated in methadone maintenance during 1969-1972. F Psychedelic Drugs 1980; 12:55-64.

38. Schiff M, Levit S, Moreno RC. Retention and illicit drug use among methadone patients in Israel: a gender comparison. Addict Behav 2007;32:2108-19.

39. Schilling RF, el-Bassel N, Schinke SP, et al. Sexual behavior, attitudes toward safer sex, and gender among a cohort of 244 recovering i.v. drug users. Int 7 Addict 1991;26:859-77.

40. Schottenfeld RS, Pakes JR, Kosten TR. Prognostic factors in Buprenorphineversus methadone-maintained patients. $\mathcal{F}$ Nerv Ment Dis 1998;186:35-43.
41. Steer RA, Kotzker E. Affective changes in male and female methadone patients. Drug Alcobol Depend 1980;5:115-22.

42. Stenbacka M, Leifman A, Romelsjo A. The impact of methadone treatment on registered convictions and arrests in HIV-positive and HIV-negative men and women with one or more treatment periods. Drug Alcohol Rev 2003;22:27-34.

43. Webber MP, Schoenbaum EE, Gourevitch MN, et al. A prospective study of HIV disease progression in female and male drug users. AIDS 1999;13:257-62.

44. Rosenthal BJ, Savoy MJ, Greene BT, et al. Drug treatment outcomes: Is sex a factor? Int 7 Addict 1979;14:45-62.

45. Heidensohn F, Gelsthorpe L. Gender and crime in Oxford handbook of criminology. London: Oxford University Press; 2012.

46. Literature review - methadone maintenance treatment. Ottawa: Health Canada 2002. Available: www.hc-sc.gc.ca/hc-ps/pubs/adp-apd/methadone/index-eng.php (accessed 2015 Aug. 11).

47. Methadone program: methadone maintenance treatment program standards and clinical guidelines. 4th ed. Toronto: The College of Physicians and Surgeons of Ontario; 2011. Available: www.cpso.on.ca/uploadedFiles/members/ MMT-Guidelines.pdf (accessed 2015 Aug. 11).

Affiliations: MiNDS Neuroscience Graduate Program (Bawor), McMaster University, Hamilton, Ont.; Population Genomics Program (Bawor, Dennis, Desai, Pare, Samaan), Chanchlani Research Centre, McMaster University, Hamilton, Ont.; Department of Clinical Epidemiology and Biostatistics (Dennis, Pare, Thabane), McMaster University, Hamilton, Ont.; Bachelor of Health Sciences Undergraduate Program (Bhalerao), McMaster University, Hamilton, Ont.; Ontario Addiction Treatment Centre (Plater, Worster, Varenbut, Daiter), Richmond Hill, Ont.; Department of Medicine (Worster, Marsh, Anglin), McMaster University, Hamilton, Ont.; Northern Ontario School of Medicine (Marsh), Sudbury, Ont.; Department of Psychiatry and Behavioural Neurosciences (Steiner, Anglin, Samaan), McMaster University, Hamilton, Ont.; Women's Health Concerns Clinic (Steiner), St. Joseph's Healthcare Hamilton, Hamilton, Ont.; Department of Obstetrics and Gynecology (Steiner), McMaster University, Hamilton, Ont.; Biostatistics Unit (Thabane), Centre for Evaluation of Medicine, Hamilton, Ont.; Peter Boris Centre for Addiction Research (Samaan), St. Joseph's Healthcare Hamilton, Hamilton, Ont.

Contributors: Monica Bawor and Zainab Samaan developed the research question and interpreted the data. Monica Bawor and Anuja Bhalerao screened articles and extracted data for the review. Monica Bawor and Brittany Dennis performed statistical analyses. Carolyn Plater, Andrew Worster, Michael Varenbut, Jeff Daiter, David Marsh, Dipika Desai and Guillaume Pare were jointly responsible for clinical interpretation and organization of the results. Rebecca Anglin and Meir Steiner were involved in data interpretation. Lehana Thabane assisted with statistical analysis and data interpretation. Monica Bawor, Brittany Dennis and Zainab Samaan drafted the manuscript, and all of the authors critically revised it. All of the authors approved the final version to be published and agreed to be guarantors of the work.

Funding: This work was funded by a grant from the Canadian Institutes of Health Research (CIHR) Drug Safety and Effectiveness Network (grant no. 126639) and an Innovation Award (grant no. 2-15311) from the Department of Psychiatry and Behavioral Neurosciences, McMaster University. Support for the study was also provided by the Chanchlani Research Centre, McMaster University, and the Peter Boris Centre for Addictions Research, St. Joseph's Healthcare Hamilton.

Acknowledgements: Monica Bawor and Brittany Dennis were supported by CIHR Intersections of Mental Health Perspectives and Addictions Research Training (IMPART) Fellowships.

Supplemental information: For reviewer comments and the original submission of this manuscript, please see www.cmajopen.ca/content/3/3/ E344/suppl/DC1 